\title{
Higgs Boson Decay in the Large $N$ Limit
}

\author{
John Morales ${ }^{1,2}$, R. Martinez ${ }^{1}$, Rafael Hurtado ${ }^{1,2}$, and Rodolfo A. Diaz ${ }^{1}$ \\ Universidad Nacional de Colombia ${ }^{1}$, Departamento de Física, Bogotá, Colombia, \\ and Centro Internacional de Física ${ }^{2}$, Bogotá, Colombia
}

Received on 13 October, 2004

\begin{abstract}
The Equivalence Theorem is commonly used to calculate perturbatively amplitudes involving gauge bosons at energy scales higher than gauge boson masses. However, when the scalar sector is strongly interacting the theory is non-perturbative. We show that the Equivalence Theorem holds in the large $N$ limit at next-to-leading order by calculating the decay widths $h \rightarrow W^{+} W^{-}$and $h \rightarrow \pi^{+} \pi^{-}$. We also show, in the same scheme of calculations, that unitarity is fulfilled for the process $h \rightarrow \pi^{+} \pi^{-}$.
\end{abstract}

\section{INTRODUCTION}

The Standard Model (SM) of the electroweak interactions, based on the $S U(2)_{L} \otimes U(1)_{Y}$ gauge symmetry [1], is a successful theory and agrees with most experimental results [2]. However, the scalar sector responsible for the symmetry breaking of the SM is not well known and it has not been tested yet. This sector gives masses to the particles of the model, fermions and gauge fields, when the scalar field has a non vanishing Vacuum Expectation Value (VEV) after the symmetry breaking. In the scalar sector a Higgs particle appears with a mass given by $m_{h}^{2}=2 \lambda v^{2}$, where $\lambda$ is the coupling constant of the self-interacting term and $v$ is the VEV $(v \approx 246$ $\mathrm{GeV}) . m_{h}$ is an unknown parameter so far.

Nevertheless, the precision tests of the SM impose strong bounds to the Higgs mass when the scalar sector is weaklycoupled. The results from LEP Electroweak Working Group analysis yield $m_{h}=114_{-45}^{+69} \mathrm{GeV}(68 \% \mathrm{CL})$ [3], and an upper limit of $m_{h}<260 \mathrm{GeV}$ with one-sided 95\% CL [3]. The direct search of the Higgs boson done at LEP gives a lower limit of $m_{h}>114.4 \mathrm{GeV}$ [3]. On the other hand, it is possible to have different models beyond the SM with a heavy Higgs with a mass lying in the $\mathrm{TeV}$ scale for a strongly interacting scalar sector. However, for this scenario to be held, the new physics contributions must cancel those ones introduced by the heavy Higgs particle at low energies [4].

If the SM is an effective theory derived from a more fundamental one, then there is an associated $\Lambda$ scale for the appearance of new physics. The use of theoretical arguments, like unitarity [5], triviality [6] and vacuum stability [7], may allow to get constraints for these two parameters $\left(\Lambda, m_{h}\right)$ [8].

The upper limit for the Higgs mass can be obtained by triviality considerations in the Higgs sector [9]. When the quartic coupling constant $\lambda$ in the scalar sector of the Higgs potential is renormalized introducing a cut-off $\Lambda$, the coupling goes to zero when $\Lambda$ goes to infinity, implying that $m_{h}$ goes to zero. This is not the case for the SM, because it needs a massive scalar particle at low energies to explain experimental results, and then the SM can be considered as an effective theory below a given energy scale. If we knew this scale we could predict the Higgs mass. Further, if the SM had a Higgs with a mass around $1 \mathrm{TeV}$, then the scalar sector would be strongly interacting and the underlying theory would become non-perturbative[10].

The amplitude for a heavy Higgs decaying into two longi- tudinally polarized gauge bosons reads [11]

$\mathcal{A}(h \rightarrow Z Z, W W) \approx \lambda\left(m_{h}\right)\left(1+2.8 \frac{\lambda\left(m_{h}\right)}{16 \pi^{2}}+62.1\left(\frac{\lambda\left(m_{h}\right)}{16 \pi^{2}}\right)^{2}\right)$.

By considering that in the perturbation expansion the $\lambda^{2}$ term must be smaller than the $\lambda$ term, it is found that $\lambda\left(m_{h}\right) \approx 7 \mathrm{im}$ plying that $m_{h} \approx 1 \mathrm{TeV}$. On the other hand, using the scattering process $W W \rightarrow Z Z$ mediated by a Higgs particle, which might be important in future collider experiments like LHC and linear colliders, the cross section for energies $\sqrt{s}>>m_{h}$ at two loops level is given by [12]

$$
\sigma(s)=\frac{1}{8 \pi s} \lambda(s)^{2}\left[1-42.65 \frac{\lambda(s)}{16 \pi^{2}}+2477.9\left(\frac{\lambda(s)}{16 \pi^{2}}\right)^{2}\right] .
$$

This cross section is negative for some values of $\lambda$ which means that the perturbative expansion breaks down. Considering that the $\lambda^{2}$ term must be smaller than the $\lambda$ term, a necessary condition to have a convergent series is $\lambda \approx 4$, in this case $m_{h} \approx 700 \mathrm{GeV}$ [13]. The above scenarios correspond to the limit between weakly-coupled and strongly-coupled scalar sectors.

In the Marciano and Willenbrock paper [14] they calculated the decays of a heavy Higgs boson up to $O\left(g^{2} m_{h}^{2} / m_{W}^{2}\right)$ in perturbation theory using the Equivalence Theorem (ET) [15], from which the amplitude with gauge bosons longitudinally polarized at energies $O\left(q^{2}>>m_{W}^{2}\right)$ is equivalent to the same amplitude but changing the corresponding longitudinal components by the would-be Goldstone bosons. For Higgs masses of the order of $m_{h} \approx 1 \mathrm{TeV}$ and $m_{h} \approx 1.3 \mathrm{TeV}$ the radiative corrections for the decay $h \rightarrow W^{+} W^{-}$are $7.3 \%$ and $12 \%$, respectively. At this scale the scalar sector is strongly-coupled and the theory is non-perturbative. It is obvious that the amplitude at next-to-leading order breaks the perturbative expansion because all Feynman rules are proportional to the Higgs mass. For strongly interacting models is necessary to use a non-perturbative method to calculate radiative corrections and get bounded amplitudes. While it has been shown that the ET holds order by order in perturbation theory, it has not been confirmed that it does in non-perturbative calculations.

Due to the importance of studying the Higgs dynamics in non-perturbative regimes, a formalism was introduced in Ref. [16] which uses Chiral Perturbation Theory $(\chi P T)$ [17]. Amplitudes are obtained as a power expansion in the energy, this 
implies that the conventional ET does not hold anymore [18]. Thus, a new formalism is necessary to have an effective theory [19].

The large $N$ limit is an alternative approach that predicts bounded positive defined amplitudes, consistent with pion dispersion [20], and useful to study the symmetry breaking of the strongly interacting sector [21]. The scalar sector of the SM can be modelled by a Linear Sigma Model $O(4)$ and then generalized to a model with $O(N+1)$ symmetry. This method has been applied to study the Higgs boson at TeV energy scales $[10,22]$. We show that the large $N$ limit can predict amplitudes that fulfill the ET and the unitarity condition at next-toleading order for the SM, with a strongly interacting scalar sector, by using the $h \rightarrow W^{+} W^{-}$and $h \rightarrow \pi^{+} \pi^{-}$processes.

In section 2 we introduce the Gauged Linear Sigma Model $O(N+1)$. In section 3 we calculate the Higgs decay widths, $h \rightarrow W^{+} W^{-}$and $h \rightarrow \pi^{+} \pi^{-}$, in the large $N$ limit and we show that the ET holds at next-to-leading order. In section 4 we show that the amplitude $h \rightarrow \pi^{+} \pi^{-}$satisfies unitarity in the large $N$ limit. In section 5 we give our conclusions.

\section{THE $O(N+1)$ MODEL}

It is well known that the Linear Sigma Model represents the symmetry breaking $O(N+1) \rightarrow O(N)$ with $N$ wouldbe Goldstone bosons which belong to the fundamental irreducible representation of the remaining symmetry $O(N)$. For the purposes of this work the would-be Goldstone bosons will be named like pions $\pi$. For a gauge invariant model under $S U(2)_{L} \otimes U(1)_{Y}$ local symmetry the large $N$ limit for the SM is defined as

$$
\mathcal{L}_{g}=\mathcal{L}_{Y M}+\left(D_{\mu} \Phi\right)^{\dagger}\left(D^{\mu} \Phi\right)-V\left(\Phi^{2}\right)
$$

with $\Phi^{\dagger}=\left(\pi_{1}, \pi_{2}, \cdots, \pi_{N}, \sigma\right)$ and $\Phi^{2}=\Phi^{\dagger} \Phi$. As usual $\mathcal{L}_{Y M}$ is the Yang-Mills Lagrangian of the SM and the covariant derivative is defined as

$$
D_{\mu} \Phi=\partial_{\mu} \Phi-i g \vec{T}^{L} \cdot \vec{W}_{\mu} \Phi+i g^{\prime} T_{3}^{R} B_{\mu} \Phi
$$

where $\vec{T}^{L}=-(i / 2) \vec{M}^{L}$ are the generators of the $S U(2)_{L}$ gauge group and $T_{3}^{R}=-(i / 2) M^{Y}$ is the generator of the $U(1)_{Y}$ 4gauge group. The $M$ matrices are given by [23]

$$
M_{i j}^{a b}=-i\left(\delta_{i}^{a} \delta_{j}^{b}-\delta_{i}^{b} \delta_{j}^{a}\right)
$$

which belong to an irreducible representation of the $O(N+1)$ Lie algebra with $i, j=1,2,3$ and $a, b=1,2, \ldots, N+1$. The matrices which belong to the adjoint representation of the $S U(2)_{L}$ Lie algebra are given by

$$
\begin{aligned}
& M_{1}^{L}=\left(\begin{array}{ccccc}
0 & 0 & 0 & \cdots & - \\
0 & 0 & - & \cdots & 0 \\
0 & + & 0 & \cdots & 0 \\
\cdots & \cdots & \cdots & \cdots & \cdots \\
\cdots & \cdots & \cdots & \cdots & \cdots \\
\cdots & \cdots & \cdots & \cdots & \cdots \\
+ & 0 & 0 & \cdots & 0
\end{array}\right) M_{2}^{L}=\left(\begin{array}{ccccc}
0 & 0 & + & \cdots & 0 \\
0 & 0 & 0 & \cdots & - \\
- & 0 & 0 & \cdots & 0 \\
\cdots & \cdots & \cdots & \cdots & \cdots \\
\cdots & \cdots & \cdots & \cdots & \cdots \\
\cdots & \cdots & \cdots & \cdots & \cdots \\
0 & + & 0 & \cdots & 0
\end{array}\right) \\
& M_{3}^{L}=\left(\begin{array}{ccccc}
0 & + & 0 & \cdots & 0 \\
- & 0 & 0 & \cdots & 0 \\
0 & 0 & 0 & \cdots & + \\
\cdots & \cdots & \cdots & \cdots & \cdots \\
\cdots & \cdots & \cdots & \cdots & \cdots \\
\cdots & \cdots & \cdots & \cdots & \cdots \\
0 & 0 & - & \cdots & 0
\end{array}\right)
\end{aligned}
$$

and, the corresponding matrix for the $U(1)_{Y}$ Lie algebra reads

$$
M^{Y}=\left(\begin{array}{ccccc}
0 & + & 0 & \cdots & 0 \\
- & 0 & 0 & \cdots & 0 \\
0 & 0 & 0 & \cdots & - \\
\cdots & \cdots & \cdots & \cdots & \cdots \\
\cdots & \cdots & \cdots & \cdots & \cdots \\
\cdots & \cdots & \cdots & \cdots & \cdots \\
0 & 0 & + & \cdots & 0
\end{array}\right)
$$

where dots represent zeros. In this form we have a global $O(N+1)$ symmetry with a local $S U(2)_{L} \otimes U(1)_{Y}$ symmetry.

The Higgs potential, invariant under $O(N+1)$, can be written as

$$
V\left(\Phi^{2}\right)=-\mu^{2} \Phi^{2}+\frac{\lambda}{4}\left(\Phi^{2}\right)^{2}
$$

Aligning the vacuum state as $\langle\phi\rangle_{0} \equiv(0, \ldots, v)$, with $\Phi^{2}=v^{2}=$ $2 \mu^{2} / \lambda$, the global symmetry $O(N+1)$ is broken to $O(N)$ and the local symmetry is broken as $S U(2)_{L} \otimes U(1)_{Y} \rightarrow U(1)_{Q}$. By defining the Higgs field as $h=\sigma-v$, we find the following expression

$$
\begin{aligned}
\mathcal{L}_{g} & =\mathcal{L}_{Y M}+\frac{1}{2}\left(D_{\mu} \pi_{a}\right)^{\dagger}\left(D^{\mu} \pi_{a}\right)+\frac{1}{2}\left(D_{\mu} h\right)^{\dagger}\left(D^{\mu} h\right) \\
& -\frac{1}{2} m_{h}^{2} h^{2}-\lambda\left(\pi^{2}+h^{2}\right)^{2}-4 \lambda v h\left(\pi^{2}+h^{2}\right) .
\end{aligned}
$$

The gauge boson masses are obtained from the kinetic term,

$$
\frac{1}{2}\left(\frac{g v}{2}\right)^{2} W_{\mu}^{a} W_{a}^{\mu}+\frac{1}{2}\left(\frac{g^{\prime} v}{2}\right)^{2} B_{\mu} B^{\mu}-\frac{g g^{\prime} v^{2}}{4} W_{\mu}^{3} B^{\mu}
$$

where the mass eigenstates are given by

$$
\begin{aligned}
W_{\mu}^{+} & =\left(W_{\mu}^{1}-i W_{\mu}^{2}\right) / \sqrt{2} \\
W_{\mu}^{-} & =\left(W_{\mu}^{1}+i W_{\mu}^{2}\right) / \sqrt{2} \\
Z_{\mu} & =\cos \theta_{W} W_{\mu}^{3}-\sin \theta_{W} B_{\mu} \\
A_{\mu} & =\sin \theta_{W} W_{\mu}^{3}+\cos \theta_{W} B_{\mu}
\end{aligned}
$$

and $\theta_{W}$ is the Weinberg angle with $\tan \theta_{W}=g^{\prime} / g$. The $W_{\mu}^{ \pm}$ fields, with $m_{W}=g v / 2 \approx 80.6 \mathrm{GeV}$ masses, are the charged gauge bosons, and the $Z_{\mu}$ field, with $m_{Z}=v\left(g^{2}+g^{\prime 2}\right)^{1 / 2} / 2 \approx$ $91.2 \mathrm{GeV}$ mass, is the weak neutral gauge boson. The $A_{\mu}$ field is the massless photon.

The Lagrangian has terms of the form $g^{2} v \partial^{\mu} \pi_{a} W_{\mu}^{a} / 4$, mixing gauge bosons with would-be Goldstone bosons, which can be cancelled by gauge fixing. We choose the Landau gauge $(\xi=0)$ because in this gauge a lot of Feynman diagrams cancel or suppress, the $\pi_{a}$ fields do not couple to the ghost fields, and their propagators are massless. The final Lagrangian can be written as 


$$
\begin{aligned}
\mathcal{L}[\pi, \vec{W}, B, h] & =-\frac{1}{2} \pi_{a} \square \pi_{a}-\frac{1}{2} h\left(\square+m_{h}^{2}\right) h-\lambda\left(\pi_{a}^{2}+h^{2}\right)^{2} \\
& -4 \lambda v h\left(\pi_{a}^{2}+h^{2}\right)-\frac{g}{2} \partial^{\mu} \pi_{1}\left(W_{\mu}^{3} \pi_{2}-W_{\mu}^{2} \pi_{3}\right) \\
& -\frac{g}{2} \partial^{\mu} \pi_{2}\left(W_{\mu}^{1} \pi_{3}-W_{\mu}^{3} \pi_{1}\right)-\frac{g}{2} \partial^{\mu} \pi_{3}\left(W_{\mu}^{2} \pi_{1}-W_{\mu}^{1} \pi_{2}\right) \\
& +g \partial^{\mu} h\left(\vec{W}_{\mu} \cdot \vec{\pi}\right)-\frac{g^{\prime}}{2}\left(\pi_{1} \partial_{\mu} \pi_{2}-\pi_{2} \partial_{\mu} \pi_{1}\right) B^{\mu}-g \partial_{\mu} h \pi_{3} B^{\mu} \\
& +\frac{1}{2} m_{W}^{2} \vec{W}_{\mu} \cdot \vec{W}^{\mu}+\frac{1}{2} m_{B}^{2} B_{\mu} B^{\mu}-m_{W} m_{B} W_{\mu}^{3} B^{\mu} \\
& +\frac{g^{2}}{8}\left(\vec{W}_{\mu} \cdot \vec{\pi}\right)\left(\vec{W}^{\mu} \cdot \vec{\pi}\right)+\frac{g^{\prime 2} v}{4} h B_{\mu} B^{\mu} \\
& +\frac{g^{\prime 2}}{8} H^{2} B_{\mu} B^{\mu}-\frac{g g^{\prime}}{4} h^{2} W_{\mu}^{3} B^{\mu}-\frac{g g^{\prime} v}{2} h W_{\mu}^{3} B^{\mu} \\
& +\frac{g^{2}}{8} h^{2} \vec{W}_{\mu} \cdot \vec{W}^{\mu}+\frac{g^{2} v}{4} h \vec{W}_{\mu} \cdot \vec{W}^{\mu}+\frac{g^{\prime 2}}{8} B_{\mu} B^{\mu} \vec{\pi} \cdot \vec{\pi} \\
& +\frac{g g^{\prime}}{4} W_{\mu}^{3} B^{\mu} \vec{\pi}^{\prime} \cdot \vec{\pi}-\frac{g g^{\prime}}{2} \pi_{3} B_{\mu}\left(W_{1}^{\mu} \pi_{1}+W_{2}^{\mu} \pi_{2}\right) \\
& +g^{\prime} m_{W} B_{\mu}\left(W_{1}^{\mu} \pi_{2}-W_{2}^{\mu} \pi_{1}\right)+\frac{g g^{\prime}}{2} B_{\mu}\left(W_{1}^{\mu} \pi_{2}-W_{2}^{\mu} \pi_{1}\right) h \\
& +\mathcal{L}_{Y M} .
\end{aligned}
$$

Thus we have a gauge theory spontaneously broken with the $\vec{\pi}=\left(\pi_{1}, \pi_{2}, \pi_{3}\right)$ fields as the would-be Goldstone bosons of the broken symmetry $S U(2)_{L} \otimes U(1)_{Y} / U(1)_{Q}$ and $\pi_{a}$ fields as the would-be Goldstone bosons of the broken global symmetry $O(N+1) / O(N)$.

The theory for the large $N$ limit makes sense when $N \rightarrow \infty$ and gives rise to finite amplitudes for different processes. To get finite amplitudes is necessary to choose appropriate parameters in the large $N$ limit. We will take the following definition

$$
\lambda \approx 1 / N
$$

in order to use perturbative expansion of the strongly interacting sector as a function of the $\lambda$ parameter. With this definition, physical masses must be finite and independent of $N$ in the large $N$ limit. From the masses

$$
\begin{aligned}
& m_{h}^{2}=2 \lambda v^{2} \approx \text { const } \\
& m_{W}^{2}=\frac{g^{2} v^{2}}{4} \approx \text { const } \\
& m_{Z}^{2}=\frac{\left(g^{2}+g^{\prime 2}\right) v^{2}}{4} \approx \text { const }
\end{aligned}
$$

we obtain for the other parameters of the model in the large $N$ limit the following values

$$
v \approx \sqrt{N}, g \approx 1 / \sqrt{N}, g^{\prime} \approx 1 / \sqrt{N}
$$

Finally, we obtain the Feynman rules necessary to calculate the decay widths for $h \rightarrow W^{+} W^{-}$and $h \rightarrow \pi^{+} \pi^{-}$in the large $N$ limit, see Fig. 1.

\section{THE HIGGS BOSON DECAY AND THE EQUIVALENCE THEOREM}

The SM in the large $N$ limit is associated with the $O(N+$ 1) $/ O(N)$ and $S U(2)_{L} \otimes U(1)_{Y} / U(1)_{Q}$ global and local symmetry breaking schemes respectively. We can calculate the amplitudes for $h \rightarrow W^{+} W^{-}$and $h \rightarrow \pi^{+} \pi^{-}$decays in order to show that the ET holds in the proposed scenario.

Feynman diagrams at tree level in this approximation are of the order of $O(g)$ or $O\left(g^{\prime}\right)$ and of the order of $O(1 / \sqrt{N})$ in the large $N$ limit . The decay widths at tree level for $h \rightarrow W^{+} W^{-}$ and $h \rightarrow \pi^{+} \pi^{-}$processes are given by

$$
\begin{aligned}
\Gamma\left(h \rightarrow W^{+} W^{-}\right) & =\frac{g^{2} m_{h}^{3}}{64 \pi m_{W}^{2}}\left[1-\frac{4 m_{W}^{2}}{m_{h}^{2}}\right]^{1 / 2}\left[1-\frac{4 m_{W}^{2}}{m_{h}^{2}}+\frac{12 m_{W}^{4}}{m_{h}^{4}}\right], \\
\Gamma\left(h \rightarrow \pi^{+} \pi^{-}\right) & =\frac{g^{2} m_{h}^{3}}{64 \pi m_{W}^{2}} .
\end{aligned}
$$



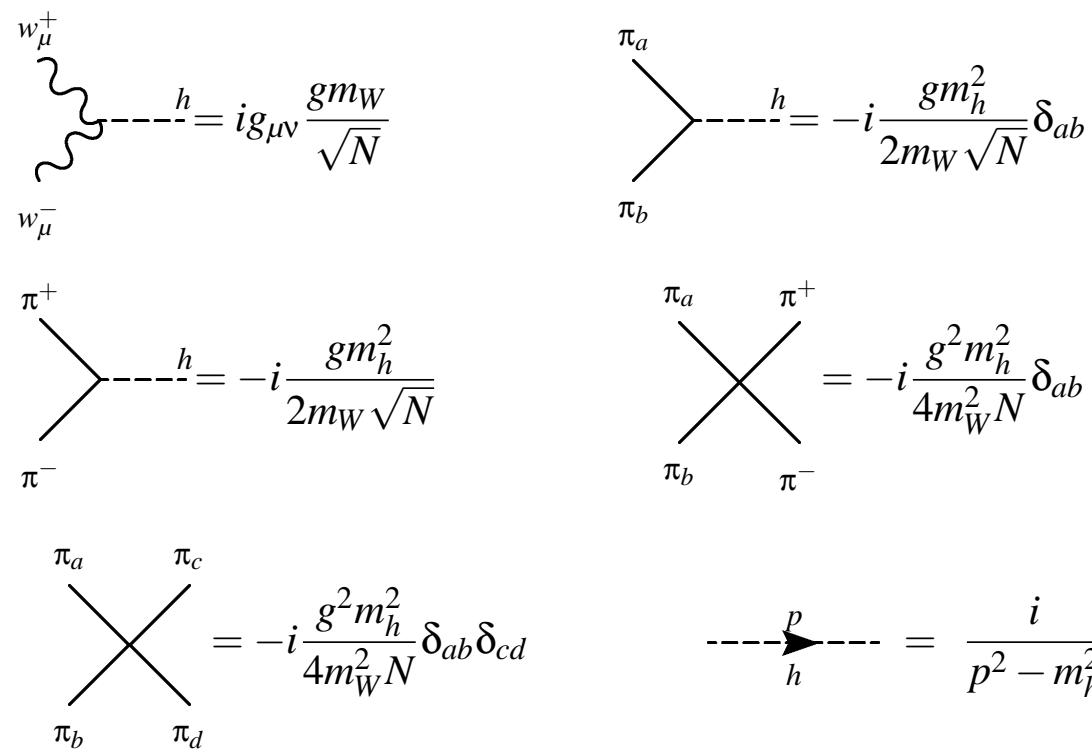

$$
\frac{p}{\pi^{ \pm}, \pi_{a}}=\frac{i}{p^{2}}
$$

Figure 1. Feynman rules in the Landau gauge for the SM in the large $N$ limit

To obtain the amplitudes at next-to-leading order is necessary to introduce the radiative corrections. First we calculate the self-energy of the scalar particle $h$, whose Feynman diagrams at next-to-leading order are shown in Fig. 2. In this case, the self-energy at one loop level with $\pi_{a}$ fields into the loops is of the order of $1 / N$ times $N$ where $N$ is the number of degrees of freedom running into the loop. Therefore, radiative corrections are of the order of one in the large $N$ limit. The same analysis can be done for the self-energy diagram with $l$ loops. It has two vertices with $h \pi \pi$ and $l-1$ vertices with four $\pi_{a}$ and is of the order of $(1 / \sqrt{N})^{2}(1 / N)^{l-1}$ times $N^{l}$ the number of pion fields running into the $l$ loops. Consequently, the self-energy diagram with $l$ loops is of the order of one in the large $N$ limit. However, the one irreducible particle function (1IP) for self-energy diagram with $W_{\mu}^{ \pm}, Z_{\mu}$ into the loop is of the order of $1 / N$, which is negligible in the large $N$ limit.

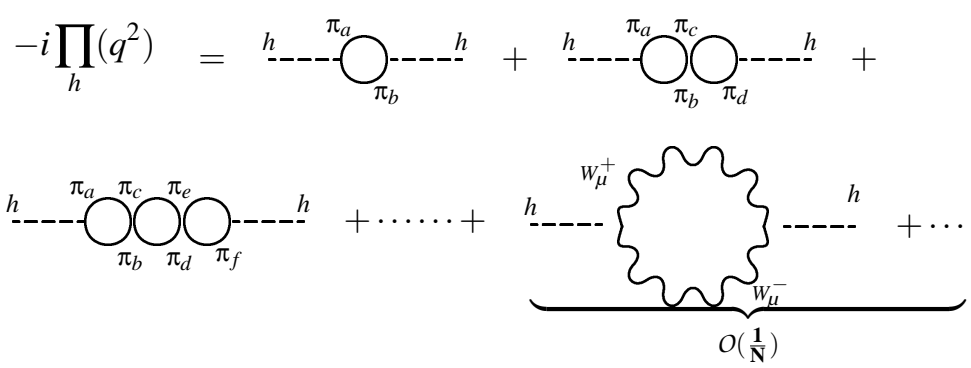

Figure 2. Next-to-leading order Feynman diagrams that contribute to the self-energy of the Higgs boson in the large $N$ limit

After doing all calculations by using dimensional regularization, with $d=4-\varepsilon$ integrals from the loops, we find (see appendix)

$$
-i \Pi_{h}\left(q^{2}\right)=\frac{\frac{g^{2} m_{h}^{4}}{8 m_{W}^{2}} I_{q}}{1-\frac{i g^{2} m_{h}^{2}}{8 m_{W}^{2}} I_{q}}
$$

with $I_{q}$ given by

$$
I_{q}=\frac{i}{16 \pi^{2}}\left(\Delta+2-\log \frac{q^{2}}{\mu^{2}}-i \pi\right)
$$

where $\Delta=2 / \varepsilon+\log 4 \pi-\gamma_{\varepsilon}$ and $\mu$ is the renormalization scale. The choice of the renormalization scale $\mu$ is arbitrary. Therefore, we shall adopt $\mu \approx 1 \mathrm{TeV}$ as a reasonable choice. We 
have taken into account that only the one irreducible particle functions are important in perturbation theory for the renormalization of parameters such as the mass and the wave function [24].

From the self-energy calculation the wave function renormalization of the Higgs boson can be obtained as

$$
Z_{h}=1+\left.\frac{\frac{g^{2}}{256 m_{W}^{2} \pi^{2} q^{2}}}{\left(1+\frac{g^{2} m_{h}^{2}}{128 m_{W}^{2} \pi^{2}}\left(\Delta+2-\log \frac{q^{2}}{\mu^{2}}-i \pi\right)\right)^{2}}\right|_{q^{2}=m_{h R}^{2}}
$$

tional to $1 / N$ and in the large $N$ limit we obtain that

$$
Z_{\pi^{ \pm}}=Z_{W_{\mu}}=Z_{Z_{\mu}}=1
$$

To calculate the Higgs decays at this order, vertex corrections have to be included as well, as shown in Fig. 3.

The contributions to $\pi^{ \pm}, W^{ \pm}$and $Z_{\mu}$ self-energies are propor-

(a)

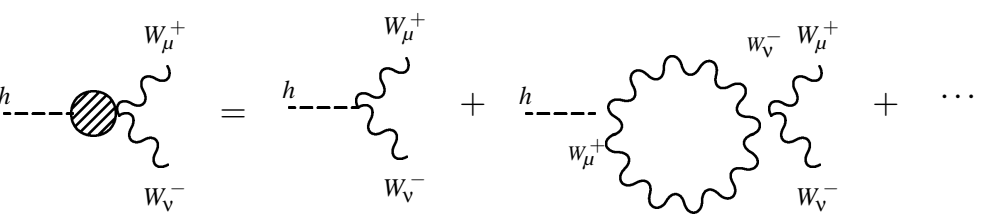

(b)
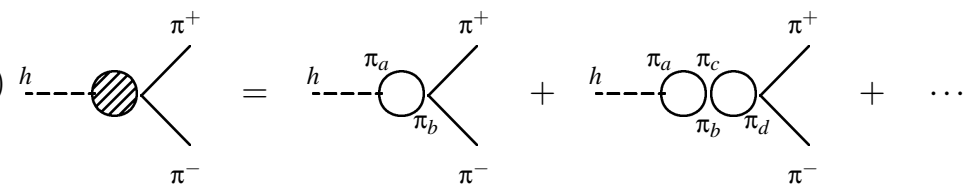
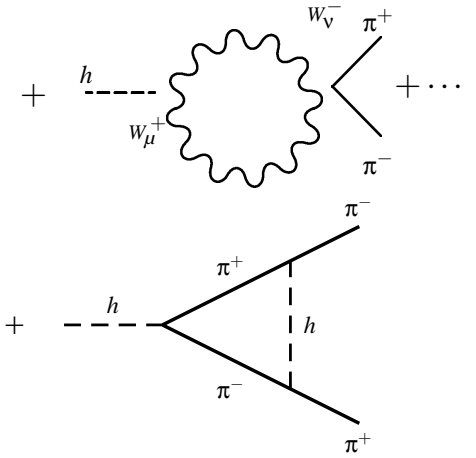

Figure 3. Feynman diagrams in the large $N$ limit which contribute to vertex interactions. (a) $h \rightarrow W^{+} W^{-},(b) h \rightarrow \pi^{+} \pi^{-}$.

The radiative corrections of $h W^{+} W^{-}$vertex displayed in Fig. 3(a) are suppressed since they are of the order of $1 / N^{2}$ becoming negligible in our approximation. The $h W^{+} W^{-}$vertex can be written at this level as

$$
\int_{W_{v}^{-}}^{W_{\mu}^{+}}=i g_{\mu v} \frac{g m_{W}}{\sqrt{N}} \text {. }
$$

For the $h \pi^{+} \pi^{-}$vertex corrections shown in Fig. 3(b), the pions into the loops give the most important contributions and can be written as

$$
\searrow_{\pi^{-}}^{\pi^{+}}=\frac{-i g}{2 m_{W} \sqrt{N}}\left[\frac{1}{\frac{1}{m_{h}^{2}}+\frac{g^{2}}{128 m_{w}^{2} \pi^{2}}\left(\Delta+2-\log \frac{q^{2}}{\mu^{2}}-i \pi\right)}\right]
$$

where the $W^{ \pm}$contributions into the loops are suppressed by a $1 / N$ factor with respect to the $\pi_{a}$ contributions. Similarly, the contribution with a higgs running into the loop is also suppressed by a $1 / N$ factor. 
The wave function renormalization of the Higgs particle Eq.(14) and the vertex radiative correction Eq. (3) diverge. To obtain finite amplitudes the Higgs mass has to be renormalized [10]

$$
\frac{1}{m_{h_{R}}^{2}} \equiv \frac{1}{m_{h}^{2}}+\frac{g^{2}(\Delta+2)}{128 \pi^{2} m_{W}^{2}} .
$$

The real part of the $Z_{h}$ function Eq.(14) is given by

$$
Z_{h}^{1 / 2}=1+\frac{g^{2} m_{h_{R}}^{2}}{16^{2} m_{W}^{2} \pi^{2}} \times \frac{\left[1-\frac{2 g^{2} m_{h_{R}}^{2}}{128 m_{W}^{2} \pi^{2}} \log \frac{m_{h_{R}}^{2}}{\mu^{2}}+\frac{g^{4} m_{h_{R}}^{4}}{8^{2} m_{W}^{4} 16^{2} \pi^{4}}\left(\log \left(\frac{m_{h_{R}}^{2}}{\mu^{2}}\right)^{2}-\pi^{2}\right)\right]}{\left|1-\frac{g^{2} m_{h_{R}}^{2}}{128 m_{W}^{2} \pi^{2}}\left(\log \frac{m_{h_{R}}^{2}}{\mu^{2}}+i \pi\right)\right|^{4}} .
$$

In the same way, the real part of the vertex correction Eq.(3) can be expressed as

$$
\int_{\pi^{-}}^{\pi^{+}}=\frac{-i g m_{h_{R}}^{2}}{2 m_{W} \sqrt{N}} \times\left[1+\frac{\frac{g^{2} m_{h_{R}}^{2}}{128 m_{W}^{2} \pi^{2}} \log \frac{m_{h_{R}}^{2}}{\mu^{2}}-\frac{g^{4} m_{h_{R}}^{4}}{8^{2} m_{W}^{4} 16^{2} \pi^{4}}\left(\log \left(\frac{m_{h_{R}}^{2}}{\mu^{2}}\right)^{2}-\pi^{2}\right)}{\left|1-\frac{g^{2} m_{h_{R}}^{2}}{128 m_{W}^{2} \pi^{2}}\left(\log \frac{m_{h_{R}}^{2}}{\mu^{2}}+i \pi\right)\right|^{2}}\right]
$$

Hence, the vertex corrections Eq.(19) multiplied by the factors of the renormalized wave functions $Z_{h}^{1 / 2} Z_{\pi}$ give rise to the $h \rightarrow \pi^{+} \pi^{-}$amplitude at next-to-leading order and can be written at $O\left(g^{2} m_{h}^{2} / m_{W}^{2}\right)$ as

$$
\begin{aligned}
\mathcal{A}\left(h \rightarrow \pi^{+} \pi^{-}\right) & =\frac{g m_{h_{R}}^{2}}{2 m_{W} \sqrt{N}}\left[1+\frac{\frac{g^{2} m_{h_{R}}^{2}}{128 m_{W}^{2} \pi^{2}} \log \frac{m_{h_{R}}^{2}}{\mu^{2}}}{\left|1-\frac{g^{2} m_{h_{R}}^{2}}{128 m_{W}^{2} \pi^{2}}\left(\log \frac{m_{h_{R}}^{2}}{\mu^{2}}+i \pi\right)\right|^{2}}\right. \\
& \left.+\frac{\frac{g^{2} m_{h_{R}}^{2}}{16^{2} m_{W}^{2} \pi^{2}}}{\left|1-\frac{g^{2} m_{h_{R}}^{2}}{128 m_{W}^{2} \pi^{2}}\left(\log \frac{m_{h_{R}}^{2}}{\mu^{2}}+i \pi\right)\right|^{4}}\right] .
\end{aligned}
$$

The same procedure is done for the $h \rightarrow W^{+} W^{-}$amplitude where the vertex corrections are multiplied by the factors of the renormalized wave functions $Z_{h}^{1 / 2} Z_{W}$, and can be written at $O\left(g^{2} m_{h}^{2} / m_{w}^{2}\right)$ as

$$
\mathcal{A}\left(h \rightarrow W^{+} W^{-}\right)=\frac{g m_{h_{R}}^{2}}{2 m_{W} \sqrt{N}}\left[1+\frac{\frac{g^{2} m_{h_{R}}^{2}}{16^{2} m_{W}^{2} \pi^{2}}}{\left|1-\frac{g^{2} m_{h_{R}}^{2}}{128 m_{W}^{2} \pi^{2}}\left(\log \frac{m_{h_{R}}^{2}}{\mu^{2}}+i \pi\right)\right|^{4}}\right] .
$$

In order to show that the ET holds for non-perturbative next-to-leading order, we calculate Higgs decays into gauge bosons and pions in the large $N$ limit. We then compare the decay widths as obtained from the decay amplitudes for $h \rightarrow \pi+\pi^{-}$and $h \rightarrow W^{+} W^{-}$in Eqs. (20) and (21) respectively. Such decay widths are given by

$$
\begin{aligned}
\Gamma\left(h \rightarrow \pi^{+} \pi^{-}\right) & =\frac{g^{2} m_{h_{R}}^{3}}{64 \pi m_{W}^{2} N}\left[1+\frac{\frac{g^{2} m_{h_{R}}^{2}}{64 \pi^{2} m_{W}^{2}} \log \frac{m_{h_{R}}^{2}}{\mu^{2}}}{\left|1-\frac{g^{2} m_{h_{R}}^{2}}{128 \pi^{2} m_{W}^{2}}\left(\log \frac{m_{h_{R}}^{2}}{\mu^{2}}+i \pi\right)\right|^{4}}\right. \\
& \left.+\frac{\frac{g^{2} m_{h_{R}}^{2}}{128 \pi^{2} m_{W}^{2}}}{\left|1-\frac{g^{2} m_{h_{R}}^{2}}{128 \pi^{2} m_{W}^{2}}\left(\log \frac{m_{h_{R}}^{2}}{\mu^{2}}+i \pi\right)\right|^{8}}\right] .
\end{aligned}
$$


and

$$
\begin{aligned}
\Gamma\left(h \rightarrow W^{+} W^{-}\right)= & \frac{g^{2} m_{h_{R}}^{3}}{64 \pi m_{W}^{2} N} \sqrt{1-4 \frac{m_{W}^{2}}{m_{h_{R}}^{2}}}\left(1-4 \frac{m_{W}^{2}}{m_{h_{R}}^{2}}+12 \frac{m_{W}^{4}}{m_{h_{R}}^{4}}\right) \\
& {\left[1+\frac{\frac{g^{2} m_{h_{R}}^{2}}{128 \pi^{2} m_{W}^{2}}}{\left|1-\frac{g^{2} m_{h_{R}}^{2}}{64 \pi^{2} m_{W}^{2}}\left(\log \frac{m_{h_{R}}^{2}}{\mu^{2}}+i \pi\right)\right|^{4}}\right] . }
\end{aligned}
$$

In Fig. 4 we display the ratio $\Gamma\left(h \rightarrow \pi^{+} \pi^{-}\right) / \Gamma\left(h \rightarrow W^{+} W^{-}\right)$as a function of the Higgs mass including next-to-leading order corrections. From this figure it can be seen that such quotient tends to one for large Higgs masses $\left(m_{h_{R}} \gtrsim 4.5 \mathrm{TeV}\right)$, showing the validity of the ET at high energies.

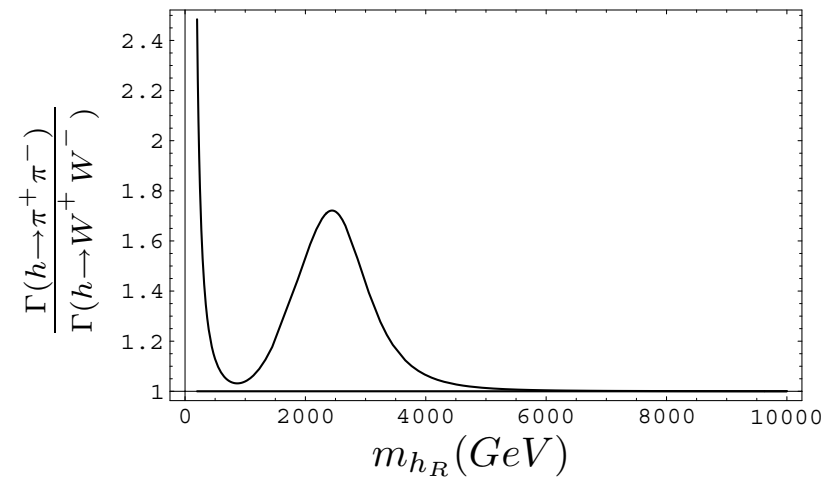

Figure 4 . The quotient $\Gamma\left(h \rightarrow \pi^{+} \pi^{-}\right) / \Gamma\left(h \rightarrow W^{+} W^{-}\right)$versus the renormalized Higgs mass, in the large $N$ limit at next-to-leading order. This Fig. shows that both decay widths tend to be equal for $m_{h_{R}} \gtrsim 4.5 \mathrm{TeV}$; showing the validity of the ET at high energies.

\section{UNITARITY IN THE LARGE $N$ LIMIT}

As a consequence of unitarity of the $S$-matrix, i. e. $S^{\dagger} S=1$, the Optical Theorem is obtained . By defining $S=1+i T$, where the $T$ is called the transition matrix, we have

$$
-i\left(T-T^{\dagger}\right)=T^{\dagger} T
$$

and since four momentum is conserved in the transition from initial state $|i\rangle$ to final state $|f\rangle$, we can always write

$$
\langle f|T| i\rangle=(2 \pi)^{4} \delta^{4}\left(p_{f}-p_{i}\right) \mathcal{T}_{f i}
$$

and

$$
\left\langle f\left|T^{\dagger}\right| i\right\rangle=\langle i|T| f\rangle^{*}(2 \pi)^{4} \delta^{4}\left(p_{f}-p_{i}\right) \mathcal{T}_{i f}^{*}
$$

Inserting a complete set of intermediate states $|q\rangle$ we find

$$
\left\langle f\left|T^{\dagger} T\right| i\right\rangle=\sum_{n}\left(\prod_{i=1}^{n} \int \frac{d^{3} q_{i}}{(2 \pi)^{3} 2 E_{i}}\right)\left\langle f\left|T^{\dagger}\right| q_{i}\right\rangle\left\langle q_{i}|T| i\right\rangle
$$

and from the identity (24) we can obtain the Cutkosky's rule[25]

$$
2 \operatorname{Im}\left(\mathcal{T}_{i f}\right)=\sum_{n}\left(\prod_{i=1}^{n} \int \frac{d^{3} q_{i}}{(2 \pi)^{3} 2 E_{i}}\right) \mathcal{T}_{f q_{i}}^{*} \mathcal{T}_{i q_{i}}(2 \pi)^{4} \delta^{4}\left(i \rightarrow \sum_{i} q_{i}\right)
$$

where the sum runs over all possible sets of intermediate states $q_{i}$.

Applying this identity to the decay $\Gamma\left(h \rightarrow \pi^{+} \pi^{-}\right)$we find

$$
\left.\left.\begin{array}{rl}
2 \operatorname{Im}\left(p-p^{+}\right. & =\int \frac{d^{3} q_{a}}{(2 \pi)^{3} 2 E_{a}} \frac{d^{3} q_{b}}{(2 \pi)^{3} 2 E_{b}}(2 \pi)^{4} \delta^{4}\left(p-q_{a}-q_{b}\right) \\
\times\left(p^{-}\right. & q_{b}
\end{array}\right)_{q_{a}}^{q_{a}}\right) .
$$

In the left-hand side of the previous equation we have the imaginary part of the product of Eq. (3) times the wave function 
$Z_{h}^{1 / 2} Z_{\pi}$, resulting

$$
2 \operatorname{Im}\left(\mathcal{A}\left(h \rightarrow \pi^{+} \pi^{-}\right)\right)=\frac{\frac{g^{3} m_{h_{R}}^{4}}{8 m_{W}^{3} 16 \pi \sqrt{N}}}{\left|1-\frac{g^{2} m_{h_{R}}^{2}}{8 m_{W}^{2} 16 \pi^{2}}\left(\log \frac{m_{h_{R}}^{2}}{\mu^{2}}+i \pi\right)\right|^{2}} .
$$

For the right-hand side, we have to multiply the amplitudes calculated in the large $N$ limit<smiles>CC#CC</smiles>

i.e.,

$$
\begin{aligned}
\mathcal{A}\left(h \rightarrow \pi_{a} \pi_{b}\right) & =\frac{g m_{h_{R}}^{2}}{2 m_{W} \sqrt{N}} \delta_{a b}\left[\frac{1}{1-\frac{g^{2} m_{h_{R}}^{2}}{8 m_{W}^{2} 16 \pi^{2}}\left(\log \frac{\left(q_{a}+q_{b}\right)^{2}}{\mu^{2}}+i \pi\right)}\right. \\
& \left.+\frac{\frac{g^{2} m_{h_{R}}^{2}}{16^{2} m_{W}^{2} \pi^{2}}}{\left(1-\frac{g^{2} m_{h_{R}}^{2}}{8 m_{W}^{2} 16 \pi^{2}}\left(\log \frac{m_{h_{R}}^{2}}{\mu^{2}}+i \pi\right)\right)^{2}}\right]
\end{aligned}
$$

by

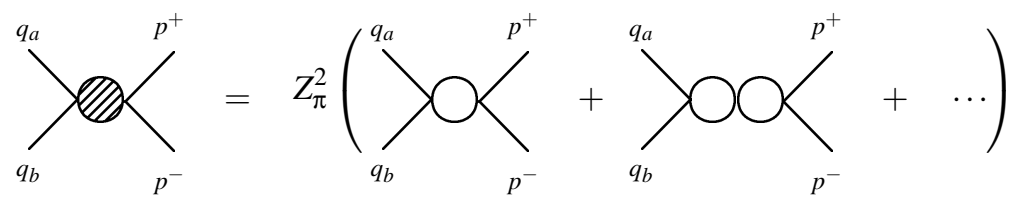

i.e.,

$$
\mathcal{A}\left(\pi_{a} \pi_{b} \rightarrow \pi^{+} \pi^{-}\right)=\frac{g^{2} m_{h_{R}}^{2}}{4 m_{W}^{2} N} \delta_{a b}\left[\frac{1}{1-\frac{g^{2} m_{h_{R}}^{2}}{8 m_{W}^{2} 16 \pi^{2}}\left(\log \frac{\left(q_{a}+q_{b}\right)^{2}}{\mu^{2}}+i \pi\right)}\right] .
$$

Then the right-hand side of Eq. (29) becomes

$$
\begin{aligned}
{\left[\mathcal{A}\left(h \rightarrow \pi_{a} \pi_{b}\right)\right]^{*}\left[\mathcal{A}\left(\pi_{a} \pi_{b} \rightarrow \pi^{+} \pi^{-}\right)\right] } & =\frac{1}{(2 \pi)^{2}} \times \frac{\frac{1}{4} \times \frac{g^{3} m_{h_{R}}^{4}}{8 m_{W}^{3} \sqrt{N}}}{\left|1-\frac{g^{2} m_{h_{R}}^{2}}{8 m_{W}^{2} 16 \pi^{2}}\left(\log \frac{\left(q_{a}+q_{b}\right)^{2}}{\mu^{2}}+i \pi\right)\right|^{2}} \\
& =\frac{1}{4} f\left(q_{a}, q_{b}\right)
\end{aligned}
$$

where $1 / 4$ is the symmetry factor for identical bosons in the final state.

From Eq. (29) we define

$$
M=\frac{1}{4 \pi^{2}} \int \frac{d^{3} q_{a}}{2 E_{a}} \frac{d^{3} q_{b}}{2 E_{b}} \delta^{4}\left(p-q_{a}-q_{b}\right) \times\left[\mathcal{A}\left(h \rightarrow \pi_{a} \pi_{b}\right)\right]^{*}\left[\mathcal{A}\left(\pi_{a} \pi_{b} \rightarrow \pi^{+} \pi^{-}\right)\right]
$$

and the integral over $q_{b}$ can be written as

$$
\int \frac{d^{3} q_{b}}{2 E_{b}}=\int_{-\infty}^{\infty} d^{4} q_{b} \delta\left(q_{a} \cdot q_{a}\right) \Theta\left(q_{b 0}\right)
$$


Integrating the four-dimensional delta function in Eq.(36) we obtain

$$
\begin{aligned}
M & =\frac{1}{16 \pi^{2}} \int \frac{\left|q_{a}\right|^{2} d\left|q_{a}\right| d \Omega}{2 E_{a}} \delta\left[\left(p-q_{a}\right)^{2}\right] \Theta\left(p_{0}-q_{a 0}\right) f\left(q_{a}, p-q_{a}\right) \\
& =\frac{1}{16 \pi^{2}} \int_{0}^{E} \frac{\left|q_{a}\right| d E_{a} d \Omega}{2} \delta\left[p^{2}-2 p \cdot q_{a}+q_{a}^{2}\right] f\left(q_{a}, p-q_{a}\right) .
\end{aligned}
$$

In the center-of-mass frame

$$
p=(E, \vec{p}), \quad q_{a}=\left(E_{a}, \vec{q}_{a}\right)=\left(E^{\prime}, \vec{q}\right), \quad q_{b}=\left(E_{b}, \vec{q}_{b}\right)=\left(E^{\prime}, \vec{q}\right)
$$

the integral in Eq. (36) can be rewritten as

$$
\begin{aligned}
M & =\frac{1}{16 \pi^{2}} \int_{0}^{E} \frac{\left|q_{a}\right| d E^{\prime} d \Omega}{2} \delta\left[E^{2}-2 E E^{\prime}-p^{2}+2 \vec{p} \cdot \vec{q}\right] f\left(q_{a}, p-q_{a}\right) \\
& =\frac{g^{3} m_{h_{R}}^{4}}{8 m_{W}^{3} 16 \pi^{2} \sqrt{N}} \frac{\left|q_{a}\right|}{\left|1-\frac{g^{2} m_{h_{R}}^{2}}{8 m_{W}^{2} 16 \pi^{2}}\left(\log \frac{p^{2}}{\mu^{2}}+i \pi\right)\right|^{2}} \frac{d \Omega}{2|-2 E|}
\end{aligned}
$$

and by using $p^{2}=m_{h_{R}}^{2}$

$$
M=\frac{\frac{g^{3} m_{h_{R}}^{4}}{8 m_{W}^{3} 16 \pi \sqrt{N}}}{\left|1-\frac{g^{2} m_{h_{R}}^{2}}{8 m_{W}^{2} 16 \pi^{2}}\left(\log \frac{m_{h_{R}}^{2}}{\mu^{2}}+i \pi\right)\right|^{2}} .
$$

Comparing equations (30) and (39) we see that the Higgs decay $\Gamma\left(h \rightarrow \pi^{+} \pi^{-}\right)$calculated in the large $N$ limit at next-toleading order fulfills the unitarity condition.

\section{CONCLUSIONS}

We have shown that non-perturbative calculations at nextto-leading order in the large $N$ limit for the case of a Higgs decaying into $W^{ \pm}$and $\pi^{ \pm}$fulfill the ET. In particular, we found that the decay widths $\Gamma\left(h \rightarrow W^{+} W^{-}\right)$and $\Gamma\left(h \rightarrow \pi^{+} \pi^{-}\right)$get values that are basically identical for heavy Higgs bosons i.e.

\section{$m_{h_{R}} \gtrsim 4.5 \mathrm{TeV}$.}

On the other hand, we have also shown that calculations in the same scheme for the Higgs decaying into pions respect unitarity. This results open the possibility to study strongly interacting systems as could be the case of the SM with a heavy Higgs boson.

\section{Acknowledgments}

We thank to Alexis Rodriguez and Marek Nowakowski for reading the manuscript and their comments. We also thank COLCIENCIAS, DIB, and DINAIN for their financial support.

\section{APPENDIX}

In this appendix we show the explicit calculation of a Feynman diagram with $l$ loops in the large $N$ limit that contributes to the Higgs boson self-energy.

$$
\begin{aligned}
\underbrace{h}_{l-\text { loop's }} \times \cdots{ }^{h} & =\frac{1}{2 l}\left[\left(\frac{-i g m_{h}^{2}}{2 m_{W} \sqrt{N}}\right)\left(-N I_{q}\right)\right] \times\left[\left(\frac{-i g^{2} m_{h}^{2}}{4 m_{W}^{2} N}\right)\right]^{l-1} \\
& \times\left(-N I_{q}\right)^{l-2} \\
& =\frac{1}{2 l}\left(-i m_{h}^{2}\right)^{l+1}\left(\frac{-g^{2} I_{q}}{4 m_{W}^{2}}\right)^{l}
\end{aligned}
$$

where $1 / 2 l$ is the symmetry factor of the diagram. The first factor corresponds to the initial and final loops times the vertices with three particles, the second factor represents the product of the $l-1$ internal vertices with four interacting fields and the last factor correspond to $l-2$ loops. Each loop 
contributes with an $N$ factor, as they have $N$ circulating pions.

[1] S. Weinberg, Phys. Lett. 19, 1264 (1967); S.L. Glashow, Nucl. Phys. B 20, 579 (1961).

[2] Particle Data Group, Phys. Rev. D 66, 1 (2002).

[3] LEP Electroweak Working Group (2004), http://lepewwg.web.cern.ch/LEPEWWG/.

[4] R. S. Chivukula and N. Evans, Phys. Lett. B 464, 244 (1999); R. S. Chivukula, N. Evans, and C. Hoelbling. Phys. Rev. Lett. 85, 511 (2000); J. Bagger, A. Falk, and M. Schwartz, Phys. Rev. Lett. 84, 1385 (2000); Michael E. Peskin, Phys. Rev. D 64, 093003 (2001).

[5] B. Lee, C. Quigg, and H. Thacker, Phys. Rev. D 16, 1519 (1977); W. Marciano, G. Valencia, and S. Willenbrock, Phys. Rev. D 40, 1725 (1989).

[6] N. Cabibbo, L. Maiani, G. Parisi, and R. Petronzio, Nucl. Phys. B 158, 295 (1979).

[7] M. Sher, Phys. Rep. 179, 273 (1989); M. Quiros, Perspectives on Higgs Physics II, Ed. G.L. Kane, World Scientific, Singapore [arXiv: hep-ph/9703412].

[8] Christopher Kolda, Hitoshi Murayama, JHEP 0007, 035 (2000).

[9] D.J.E Callaway, Phys. Rep. 167, 241 (1988); T. Hambye and K. Riesselmann, Phys. Rev. D 55, 7255 (1997); J.S. Lee and J.K. Kim, Phys. Rev. D 53, 6689 (1996).

[10] A. Dobado, J. Morales, J.R. Pelaez, and M.T. Urdiales, Phys. Lett. B 387, 563 (1996); A. Ghinculov, T. Binoth, and J.J. van der Bij, Phys. Rev. D 57, 1487 (1998).

[11] A. Ghinculov, Nucl. Phys. B 455, 21 (1995); A. Frink et. al., Phys. Rev. D 54, 4548 (1996).

[12] P. Maher, L. Durand, and K. Riesselmann, Phys. Rev. D 48, 1061 (1993); 52, 553 (1995); K. Riesselmann, Phys. Rev. D 53, 6626 (1996).

[13] U. Nierste and K. Riesselmann, Phys. Rev. D 53, 6638 (1996).

[14] W.J. Marciano and S.S.D. Willenbrock Phys. Rev. D 37, 2509 (1988).
[15] B.W. Lee, C. Quigg, and H. Thacker Phys. Rev. D 16, 1519 (1977). J.M. Cornwall, D.N. Levin, and G. Tiktopoulus, Phys. Rev. D 10, 1145 (1974). M.S. Chanowitz and M.K. Gallaird Nucl. Phys. B 261, 379 (1985). G.K. Gounaris, R. Kogerler, and H. Neufeld, Phys. Rev. D 34, 3257 (1986). H.J. He and W. Kilgore, Phys. Rev. D 55, 1515 (1997).

[16] A. Dobado and M. Herrero, Phys. Lett. B 228, 495 (1989). J. Donoghue and C. Ramirez, Phys. Lett. B 234, 361 (1990).

[17] J. Gasser and H. Leutwyler, Ann. of Phys. 158, 142 (1984). S. Weinberg, Phys. Rev. Lett. 19, 1264 (1967); M.S. Chanowitz, M. Golden, and H. Georgi, Phys. Rev. D 36, 1490 (1987); A. Longhitano, Phys. Rev. D 22, 1166 (1980); Nucl. Phys. B 188, 118 (1981).

[18] H. Veltman, Phys. Rev. D 41, 264 (1990).

[19] A. Dobado, J.R. Pelaez, and M. T. Urdiales, Phys. Rev. D 56, 7133 (1997).

[20] A. Dobado, A. Lopez, and J. Morales, Il Nuovo Cimento A 108, 335 (1995); A. Dobado and J. Morales, Phys. Rev. D 52, (1995) 2878.

[21] S. Coleman, R. Jackiw, and H. D. Politzer, Phys. Rev. D 10 2491 (1974); Dolan and Jackiw, Phys. Rev. D 9, 3320 (1974); M. Kobayashi and T. Kugo, Prog. Theor. Phys. 54, 1537 (1975); W. Bardeen and M. Moshe, Phys. Rev. D 28, 1372 (1983).

[22] R.Casalbuoni, D. Dominici, and R. Gatto, Phys. Lett. B 147, 419 (1984); M.B. Einhorn, Nucl. Phys. B 246, 75 (1984); A. Dobado and J.R. Pelaez, Phys. Lett. B 286, 136 (1992).

[23] H. Georgi, Lie Algebras in Particle Physics, Addison Wesley, Frontiers in Physics. New York 1982.

[24] M. E. Peskin, D. V. Schroeder, An introduction to quantum field theory, Westview Press (1995), Chap. 7.

[25] R. E. Cutkosky, J. Math. Phys. 1, 429 (1960). 